\title{
The occurrence of polyprenols in seeds and leaves of woody plants
}

\author{
Marcin Chouda ${ }^{1}$ and Wiesław Jankowski ${ }^{2 凶}$ \\ ${ }^{1}$ Agricultural University, SGGW, Warszawa, Poland, ${ }^{2}$ Institute of Biochemistry and \\ Biophysics, Polish Academy of Sciences, Warszawa, Poland
}

Received: 17 January, 2005; accepetd: 15 March, 2005

Key words: polyprenols, dolichols, seed lipids, leaf lipids

\begin{abstract}
The contents of the heterogenous group of polyisoprenoids was found about two orders of magnitude lower in seeds than the amount of polyprenols and/or their carboxylic esters accumulated during vegetation season in leaves. In contrast to leaves, no seeds were found containing more than $0.5 \mathrm{mg}$ of these lipids per gram of dry tissue. Almost $50 \%$ had less than $0.01 \mathrm{mg} / \mathrm{g}$ - the amount which is the limit of detection by the procedure used in this work. In gymnosperms (10 representatives of Cupressaceae, Pinaceae and Taxaceae) the polyprenol spectra in seeds and in needles were similar. In angiosperms (25 representatives of 13 botanical families) the polyisoprenoid mixture in seeds resembled the minor, additional subfamily found in leaves.
\end{abstract}

Terpenoids (syn., isoprenoids), biosynthesised from isopentenyl pyrophosphate are the largest group of natural compounds. The number of known structures, doubling every decade, could soon reach $10^{5}$. Among them, polyisoprenoids i.e. uncyclized $=$ linear polymers of the $\mathrm{C}_{5}$ unit have been very often found in the examined tissues.

The occurrence of long chain poly-cis-prenols accumulating in leaves in the majority of the hundreds plant species analysed has been well documented (Swieżewska et al., 1994; Jankowski et al., 1994). Numerous species of Spermatophyta belonging to: Asetraceae, Euphorbiaceae, Laureaceae, Magnoliaceae, Moraceae, Pinaceae, Rosaceae, Sapindaceae and Tiliaceae accumulate in leaves family(ies) of polyprenols, of chain length $\mathrm{C}_{35-500 \text {, in }}$ amounts approaching approx.i $5 \%$ of dry weight. However, in other plant organs the amounts of polyprenols were found to be rather low, with exception of wood of Betula

\footnotetext{
${ }^{凶}$ Correspondence to: W. Jankowski, Institute of Biochemistry and Biophysics, Polish Academy of Sciences, A. Pawińskiego 5a, 02-106 Warszawa, Poland; phone: (48) 592 3500; fax: (48 22) 6584 4636; email: jankowski@ibb.waw.pl
}

Abbreviation: TLC, thin-layer chromatography. 
verrucosa (Lindgren, 1965), and it seems that only in photosynthetic tissues distinct accumulation of polyprenols and perhaps their biosynthesis takes place. Recent isolation of new polyisoprenoid structures differing from the previously known ones in the number and configuration of the $\mathrm{C}=\mathrm{C}$ double bonds indicates considerable heterogeneity of these lipids, obvious in extracts from leaves of plants containing them in moderate amounts (Suga et al., 1989). As shown in the case of the all-trans-rubber-producing Eucommia ulmoides various plant organs (leaves, roots, seed coat) exhibit structural diversity of polyprenoids (Bamba et al., 2001), i.e. both poly-cisand poly-trans-prenols of different chain length were observed.

\section{Polyprenols}
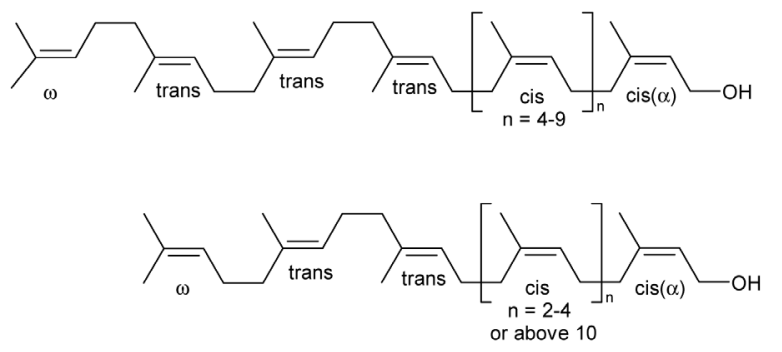

Dolichols

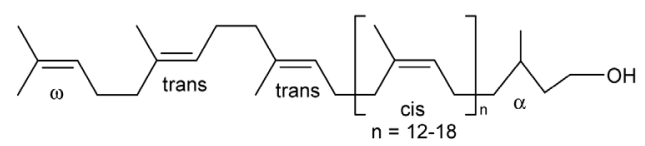

Scheme 1. Structures of polyprenols and dolichols.

The role of phoshopolyisoprenoids as universal coenzymes and carriers of sugar residues for glycosylation in a variety of biosynthetic reactions is well documented (Hemming, 1992). However, only recently the role of the dominating free polyisoprenols and their carboxylic esters as components of the antioxidant machinery located in the cell membranes has been uncovered (Bergamini et al., 2004). The protective role of polyisoprenoids against reactive oxygen and nitrogen species has induced interest of pharmaceutical industry with these lipids. There- fore, finding a source of easily obtainable polyisoprenoids of high purity should be of both scientific and economic interest. Ravi et al. (1984) have shown for a limited number of dicotyledonous species that seeds contained low amounts of dihydropolyprenols (dolichols), and mixtures of equal amounts of polyprenols and dolichols were found in seeds of monocotyledons. To extend those data we compared the content and the type of polyisoprenoids in seeds of other easily available species of large evolutionary distance, known to accumulate polyprenols in leaves. The spectra of polyprenoids from seeds and needles of gymnosperms were found to be similar whereas a complex mixture of polyisoprenoids was found in the seeds of angiosperms. The mixture consisted of a family of polyprenols resembling the minor, additional subfamily of polyprenols found in leaves and of a family of dolichols. The range of chain lengths of those polyprenols and dolichols was similar.

\section{METHODS}

Materials. Leaves of angiosperm plants were collected in September 1996 in the Arboretum of the Institute of Dendrology, Polish Academy of Sciences in Kórnik, and in the Botanical Garden of the Polish Academy of Sciences in Powsin. Seeds were from the Arboretum of the Institute of Dendrology in Kórnik; most of them were collected in 1996. In a few cases they were from earlier collections. Chromatographic materials were from Merck, Darmstadt (Germany); all solvents and other chemicals were of analytical grade from POCh Gliwice (Poland).

Preparation of samples for analysis. Leaves harvested to paper envelopes were immediately chilled, then put unfrozen into lyophilization jar within a few hours after harvesting and dehydrated in vacuum for $36 \mathrm{~h}$. Seeds were dehydrated without the external parts. From the lyophilized material $1 \mathrm{~g}$ samples were taken for homogenization in $10 \mathrm{ml}$ 
of hexane using a Janke \& Kunkel Ultra turrax T25 homogenizer at $24000 \mathrm{rev} / \mathrm{min}$. for $2 \mathrm{~min}$. The homogenates were transferred into $0.8 \times 6 \mathrm{~cm}$ Pasteur pipettes clogged with a piece of glass wool. After filtering the solvent the residue was leached with $10 \mathrm{ml}$ of acetone and finally with $10 \mathrm{ml}$ of chloroform/methanol, 2:1 (v/v) mixture.

Study of lipid substances. The three extracts and the lipids fractions were analysed by thin-layer chromatography (TLC) on Kiesel gel plates in solvent A (hexane/toluene, 2:1, v/v), solvent B (ethyl acetate/toluene, 1:19, v/v) and solvent $\mathrm{C}$ (chloroform/methanol/water, 65:25:4, by vol.). For checking the type of polyprenols RP-18 plates and acetone/methanol, 9:1, v/v (solvent D) were used.

The presence of spots of polyisoprenoids was revealed with the aid of anisaldehyde spray reagent (5\% anisaldehyde and 5\% $\mathrm{H}_{2} \mathrm{SO}_{4}$ in ethanol) and subsequent heating at $100^{\circ} \mathrm{C}$. The characteristic greenish-gray colour of a spot is a useful indicator of the presence of a polyisoprenoid on the plate. For detecting polyprenols on RP-18 plates the spots were visualized with iodine vapours and, after evaporation of iodine, with the anisaldehyde spray.

The quantities of polyprenols and their esters were measured by three methods:

-1. Comparison with the area of two spots similar in size of increasing amounts of cochromatographed standards - the neighbour points on both sides within the calibration curve.

2. Measuring the weight (with the accuracy $0.1 \mathrm{mg}$ or $0.001 \mathrm{mg}$ ) of purified polyisoprenoid preparations kept previously overnight under vacuum in an exicator over granulated paraffin wax applied as a trap for residual hydrophobic solvents.

3. Comparison of the area (calculated with the use of software Maxima 820-Waters, U.S.A.) of chromatographic peaks of isoprenologues separated on reversed phase HPLC column with the area of the peak of an internal standard - isoprenologue of different chain length therefore different elution time. The discrepancies between method 2 and 3 were below $25 \%$. Saponification of lipids was performed according to Stone et al. (1967) by treating the lipid sample isolated from $1 \mathrm{~g}$ of material dissolved in $1 \mathrm{ml}$ of benzene with $1 \mathrm{ml}$ of $1 \mathrm{M}$ $\mathrm{KOH}$ in $85 \%$ aqueous ethanol for $1 \mathrm{~h}$ at $90^{\circ} \mathrm{C}$. The unsaponifiable lipids were isolated by three subsequent extraction of the hydrolysate diluted with $1 \mathrm{ml}$ of water with $3 \mathrm{ml}$ of ethyl ether, 3 times. The combined ether extract was washed with $5 \mathrm{ml}$ of water. After evaporating the solvent with stream of $\mathrm{N}_{2}$ the unsaponifiable lipids were dissolved in $0.3 \mathrm{ml}$ of hexane and separated on a Lichroprep Si60 column packed into $0.8 \times 6 \mathrm{~cm}$ Pasteur pipettes clogged with a piece of glass wool.

The lipid sample was applied in hexane and the column eluted with two $5 \mathrm{ml}$ portions of $5 \%$ ethyl ether in hexane (fractions 1 and 2) and with two $5 \mathrm{ml}$ portions of $12 \%$ ethyl ether in hexane (fractions 3 and 4). The presence of polyprenols in the four fractions was checked by TLC (solvents A and B) and the fractions containing free polyisoprenoid alcohols were taken for further analysis.

HPLC of polyisoprenoid alcohols was performed on a Nucleosil 120-C18, $5 \mu \mathrm{m}$ column $4.6 \times 250 \mathrm{~mm}$ using a Waters apparatus and a linear gradient from $20 \%$ to $50 \%$ of a mixture of hexane/isopropanol $-7: 3$ in a mixture of methanol/isopropanol/water $(12: 8: 1$, by vol.).

Polyisoprenoid standards serving as reference substance came from the local „Collection of Polyprenols" (Institute of Biochemistry and Biophysics, PAS, Warszawa, Poland). The other test lipid substances used (fatty acids, triglyceride and phospholipid mixtures) were prepared from sunflower oil or were from Larodan Fine Chemicals (AB, Malmo, Sweden). 


\section{RESULTS}

\section{The lipid substances in seeds of gymno- sperms}

Free polyisoprenoid alcohols and their carboxylic esters were present exclusively in the first-hexane extract, but they could be observed by TLC only after chromatography on silica (the penultimate step on Scheme 2). The contents of substances present in the acetone and chloroform-methanol fractions were estimated gravimetrically and the lipids were investigated only summarily by TLC. In the acetone extracts from oily seeds e.g. $P$. cembra some triglycerides could be observed whereas the largest amounts of ste- roids are present in the extract from $T$. orientalis. The main components of the chloroform-methanol extract were phospholipids: phosphatidylcholine approx. 50\%, phosphatidylserine approx. 10\%, phosphatidylethanolamine approx. $10 \%$ and cardiolipine approx. $5 \%$, (TLC on silica gel, solvent C).

In Table 1 are shown the amounts of lipids from seeds of ten species of gymnosperms extracted according to Scheme 2. Most of the seed lipids were extracted with hexane and after removal of the dominating triglycerides (after alkaline hydrolysis) gave the fraction of unsaponifiable lipids. The last column of Table 1 shows the amount of polyprenols present in fraction 3 (eluted with $12 \%$ ethyl eter) from the silica gel column, estimated by com-

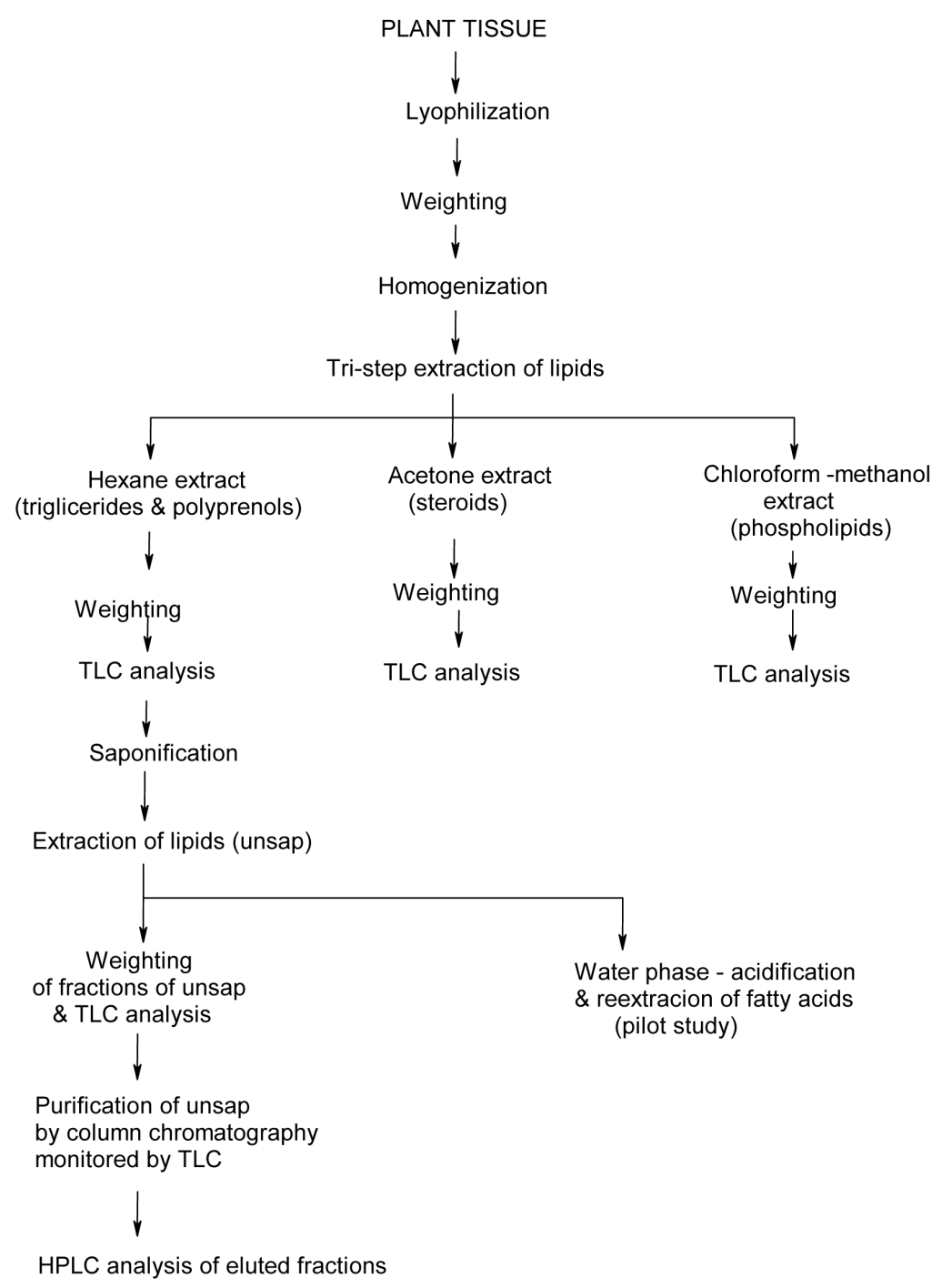

Scheme 2. Analytical procedure applied to the seeds.

unsap., unsaponifiable lipids. 
paring the intensity and size of the TLC spot with that obtained for a known amount of pure pentadecaprenol cochromatographed on the same silica gel TLC plate, solvent system $\mathrm{B}$, detection with anisaldhyde reagent (Stone et al., 1967). dles are up to 100 times higher. Although there is some similarity in the spectra of T. nucifera to that of T. baccata (Fig. 1g), both Taxaceae, apparently the most complex spectrum of polyisoprenoids was observed in extracts from $T$. baccata where the first family

Table 1. The amounts (\% of dry weight) of lipid substances extracted from seeds of gymnosperms

\begin{tabular}{lllllll}
\hline Species & Hexane $^{\mathrm{a}}$ & Acetone $^{\mathrm{a}}$ & Chl-Met $^{\mathrm{a}}$ & Total $^{\mathrm{a}}$ & UNSAP $^{\mathrm{a}}$ & Isoprenoids $^{\mathrm{b}}$ \\
\hline Abies alba & 24.66 & 6.61 & 2.02 & 33.29 & 2.251 & Not detect \\
Cedrus atlantica & 35.19 & 3.86 & 1.75 & 40.80 & 5.110 & Not detect \\
Larix decidua & 7.00 & 0.69 & 1.92 & 9.61 & 0.368 & 0.001 \\
Picea abies & 28.04 & 1.38 & 1.50 & 30.92 & 0.364 & 0.010 \\
Pinus cembra & 53.85 & 1.61 & 3.27 & 58.73 & 0.337 & 0.012 \\
Pinus nigra & 26.11 & 2.73 & 1.56 & 30.40 & 0.437 & 0.014 \\
Pinus silvestris & 27.33 & 1.28 & 1.94 & 30.55 & 0.252 & 0.015 \\
Taxus baccata & 25.81 & 4.63 & 0.78 & 31.22 & 0.117 & 0.003 \\
Thuja orientalis & 11.23 & 1.34 & 1.61 & 14.18 & 0.301 & 0.005 \\
Torreya nucifera & 45.72 & 1.49 & 2.05 & 49.26 & 0.331 & 0.055 \\
\hline
\end{tabular}

${ }^{\mathrm{a}}$ The content of lipids in: hexane, acetone, chloroform/methanol (2:1, v/v), extracts and unsaponifiable lipid (UNSAP) fractions were estimated gravimetrically. ${ }^{b}$ Contents of polyisoprenoids calculated from the area of chromatographic spot.

Seeds of C. atlantica and A. alba contain up to 20 times more unsaponifiable lipids than seeds of other gymnosperm plants studied. Most of these substances migrate on silica gel TLC plates in solvent B as two distinctive spots $\left(R_{\mathrm{F}}=0.42\right.$ and 0.31$)$ slightly slower than polyisoprenoids $\left(R_{\mathrm{F}}=0.48-0.54\right.$, solvent $\left.\mathrm{B}\right)$ but the colour of their derivatives with anisaldehyde differs from that of polyisoprenoids or cholesterol and $\beta$-sitosterol $\left(R_{\mathrm{F}}=0.27\right)$. These interesting lipids dominate the unsaponifiable lipids and they interfere with their isolation and identification (Wellburn \& Hemming, 1966).

In Table 2 and Fig. 1 are shown polyisoprenoid spectra in seeds and needles of gymnosperms. The spectra of polyisoprenoids in seeds of all three Pinus species are the same and in the seeds and needles of Pinaceae are very similar and simple $\left(\mathrm{C}_{80}\right.$-prenoid dominates, Fig. 1f) although their contents in nee- with $\mathrm{C}_{75}$ dominant overlaps the second where $\mathrm{C}_{80}$ and $\mathrm{C}_{110}$ polyisoprenoids dominate. Seeds of T. nucifera (Fig. 1i, Fig. 2 lower line) contain several times more polyisoprenoids than other examined seeds, however, it is only approx. $5 \%$ of the amounts present in needles (Fig. 2 upper line). The mixture of polyisoprenoids from seeds of $T$. orientalis (Cupressaceae) is jointly dominated by both $\alpha$-un, and $\alpha$-saturated types of polyisoprenoids with chain length $\mathrm{C}_{85}$ and $\mathrm{C}_{90}$ (Fig. 1h). Apart from the presented taxonomic similarities the occurrence of both types of polyisoprenoids in the seed extracts is indicated by unsymmetrical ( $P$. nigra and T. nucifera, Figs. If and 1i) or split (T. baccata and T. orientalis, Figs. $1 \mathrm{~g}$ and $1 \mathrm{~h}$ ) peaks on the chromatograms. The ratio of lipids migrating like dolichols to those which behave like polyprenols is different: $P$. abies and $P$. nigra $=$ $0.2, T$. nucifera $<0.1, T$. orientalis $=2.2$. 
Table 2. Comparison of polyisoprenoids in seeds and needles of gymnosperms

\begin{tabular}{|c|c|c|}
\hline Species & Prenologues (number of isopren units) ${ }^{a, b}$ & Contents (\% of dry weight) \\
\hline \multicolumn{3}{|c|}{ Larix decidua } \\
\hline Seeds & $15, \mathbf{1 6}, 17,18$ & 0.001 \\
\hline Needles & $14,15, \mathbf{1 6}, 17,18,19$ & 0.11 \\
\hline \multicolumn{3}{|c|}{ Picea abies } \\
\hline Seeds & $14,15, \mathbf{1 6}, 17,18$ & 0.010 \\
\hline Needles & $14, \mathbf{1 5}, \mathbf{1 6}, 17,18$ & 0.55 \\
\hline \multicolumn{3}{|c|}{ Pinus nigra } \\
\hline Seeds & $12,13,14,15, \mathbf{1 6}, 17,18,19$ & 0.010 \\
\hline Needles & $13,14,15, \mathbf{1 6}, 17,18$ & 0.22 \\
\hline \multicolumn{3}{|c|}{ Taxus baccata } \\
\hline Seeds & $12,13,14, \mathbf{1 5}, 16,17, \mathbf{1 8}, 19,20,21, \mathbf{2 2}, 23,24,25$ & 0.003 \\
\hline Needles & $16,17, \mathbf{1 8}, 19,20,21, \mathbf{2 2}, 23,24$ & 0.37 \\
\hline \multicolumn{3}{|c|}{ Thuja orientalis } \\
\hline Seeds & $15,16, \mathbf{1 7}, \mathbf{1 8}, 19,20$ & 0.005 \\
\hline Needles & $14,15, \mathbf{1 6}, \mathbf{1 7}, 18,19,20,21,22,23$ & 0.21 \\
\hline \multicolumn{3}{|c|}{ Torreya nucifera } \\
\hline Seeds & $14,15,16,17, \mathbf{1 8}, 19,20,21, \mathbf{2 2}, 23,24,25$ & 0.055 \\
\hline Needles & $14,15,16, \mathbf{1 7}, \mathbf{1 8}, 19,20,21, \mathbf{2 2}, 23,24$ & 1.10 \\
\hline
\end{tabular}

${ }^{\mathrm{a}}$ The numbers refer to the isoprene units of polyisoprenoid alcohols. ${ }^{\mathrm{b}}$ Dominating isoprenologues are indicated in bold. ${ }^{\mathrm{c}}$ Contents calcutated from HPLC peak areas.

\section{The lipid substances in seeds of angio-} sperms

Table 3, similary to Table 1 shows the weights of the lipid extracts obtained according to Scheme 2 from seeds of angiosperms. Almost half of the analysed seeds contained, in $1 \mathrm{~g}$ sample, polyisoprenoids in amounts to small to be identified and estimated during the applied procedure.

Representative HPLC spectra of polyisoprenoids isolated from seeds and leaves of five angiosperms according to Scheme 2, are presented in Table 4 and Fig. 1. Due to the low amount of polyisoprenoids only the main dominating component of the mixture could be recognised as a polyprenol or dolichol. The seeds contain at least 2 times more dolichols than polyprenols. The ratio of lipids migrating like dolichols to those which behave like polyprenols is for A. rubrum =1.8 (Fig. 1c), $H$. rhamnoides $=2.7$ (Fig. 1e), $F$. silvatica $=2.9$
(Fig. 1d), T. cordata $=4.3$ (Fig. 1b), Q. rubra $=$ 5.1 (Fig. 1a).

\section{DISCUSSION}

The presented results are in support of the universal occurrence of small amounts of a complex mixture of polyisoprenoids in seeds. In spite of the accumulation of considerable amounts of polyprenols being common in plants it was found that dolichyl- and not polyprenyl-phosphates function as sugar lipid carriers in glycoprotein biosynthesis in plants (Elbein, 1979). At the same time it was noticed that occasionally, preparations of $\mathrm{C}_{85-105}$ dolichols extracted from mammalian organs contain also up to $20 \%$ of $\mathrm{C}_{55}$ dolichol or $\mathrm{C}_{50}$ polyprenol, i.e. unusually short chain length polyisoprenoids for animal tissues (Mańkowski et al., 1976; Radomińska-Pyrek et al., 1979). 


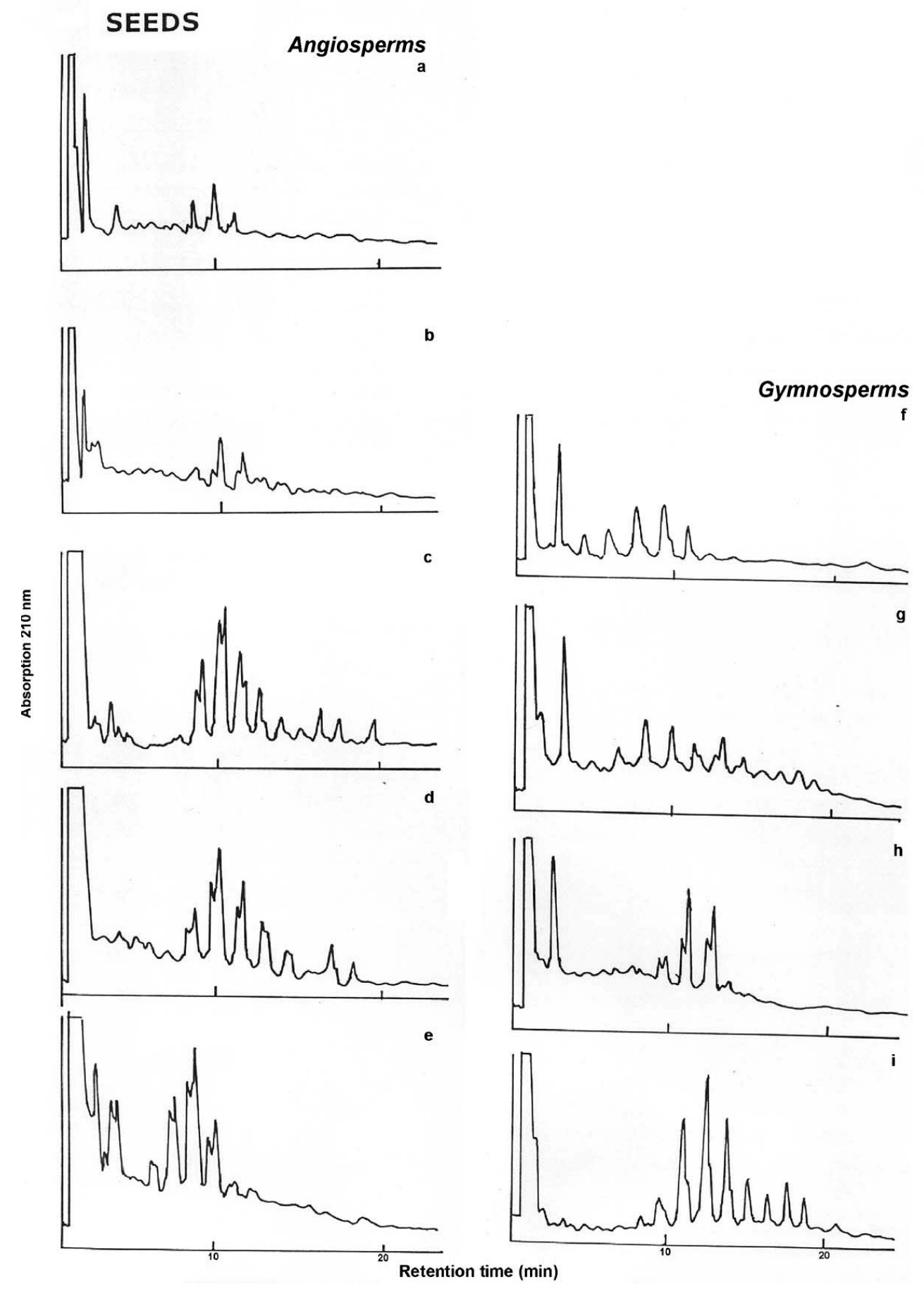

Figure 1. Polyisoprenoid spectra from seeds.

a, Q. rubra; b, T. cordata; c, A. rubrum; e, H. rhamnoides; f, P. nigra; g, T. baccata; h, T. orientalis; i, T. nucifera.

Recently, multiple polyisoprenoids of different structure are observed in individual plant tissues (Rezanka \& Votruba, 2001; Bamba et al., 2001; Skorupińska-Tudek et al., 2003; Bamba et. al., 2004) thanks to the application of rapidly developing research techniques es- pecially high performance liquid and supercritical fluid chromatography combined with mass spectrometry. Their use allows for more exact physical separation of polyisoprenoids or for estimation of the content of different polyisoprenoids present in the same chro- 
Table 3. The amounts (\% of dry weight ) of lipid substances extracted from seeds of angiosperms

\begin{tabular}{lllllll}
\hline Species & Hexane $^{\mathrm{a}}$ & Acetone $^{\mathrm{a}}$ & Chl-Met $^{\mathrm{a}}$ & Total $^{\mathrm{a}}$ & UNSAP $^{\mathrm{a}}$ & Isoprenoids $^{\mathrm{b}}$ \\
\hline Acer circinatum & 10.14 & 2.43 & 2.30 & 14.87 & 0.36 & 0.003 \\
Acer ginnala & 22.09 & 12.14 & 2.39 & 36.62 & 0.52 & 0.025 \\
Acer pseudoplatanum & 9.79 & 1.21 & 1.76 & 12.76 & 0.43 & 0.003 \\
Acer rubrum & 15.00 & 11.84 & 1.96 & 28.80 & 0.38 & 0.002 \\
Acer saccharinum & 2.51 & 1.80 & 1.80 & 6.11 & 0.15 & Not detect \\
Carpinus betulus & 4.74 & 0.69 & 1.13 & 6.56 & 0.24 & Not detect \\
Corylus colurna & 55.42 & 0.78 & 1.88 & 58.08 & 0.37 & Not detect \\
Cotonoaster horizontalis & 3.22 & 1.31 & 0.90 & 5.43 & 0.22 & Not detect \\
Cotonoaster multiflora & 4.11 & 0.93 & 1.12 & 6.16 & 0.18 & Not detect \\
Crategus submollis & 2.34 & 0.60 & 1.04 & 3.98 & 0.16 & Not detect \\
Fagus silvatica & 26.54 & 1.11 & 1.28 & 28.93 & 0.21 & 0.003 \\
Hippophe rhamnoides & 9.73 & 4.07 & 1.59 & 15.39 & 0.34 & 0.003 \\
Laburnum anagyroides & 7.23 & 1.16 & 3.16 & 11.55 & 0.18 & 0.013 \\
Prunus avium & 36.28 & 1.10 & 2.74 & 40.12 & 0.31 & 0.007 \\
Quercus rubra & 14.12 & 9.24 & 3.16 & 26.52 & 0.29 & 0.006 \\
Rosa canina & 9.20 & 0.89 & 1.72 & 11.81 & 0.27 & Not detect \\
Sambucus nigra & 30.16 & 0.64 & 1.21 & 32.01 & 0.34 & Not detect \\
Shepherdia argentea & 9.83 & 1.65 & 2.23 & 13.71 & 0.38 & Not detect \\
Staphylea trifoliata & 21.99 & 0.54 & 1.54 & 24.07 & 0.28 & 0.003 \\
Symphoricarpos albus & 20.20 & 0.54 & 1.28 & 22.02 & 0.33 & Not detect \\
Tilia cordata & 25.47 & 0.60 & 1.84 & 27.91 & 0.27 & 0.002 \\
Ulmus glabra & 36.36 & 0.84 & 2.50 & 39.70 & 0.40 & 0.005 \\
Ulmus laevis & 33.53 & 0.56 & 2.86 & 36.95 & 0.35 & 0.005 \\
Viburnum lantana & 12.93 & 3.32 & 5.04 & 21.29 & 0.56 & Not detect \\
Viburnum opulus & 10.93 & 5.53 & 6.00 & 22.46 & 0.55 & Not detect \\
\hline & & & & & &
\end{tabular}

${ }^{\mathrm{a}}$ The content of lipids in: hexane, acetone, chloroform/methanol (2 :1, v/v) extracts and unsaponifiable lipid (UNSAP) fractions were estimated gravimetrically. ${ }^{b}$ Contents of polyisoprenoids calculated from the area of chromatographic spot.

matographic peak. The results obtained by the application of these methods generally confirm the universal occurrence of heterogeneity of these lipids encountered repeatedly in the past (Mankowski et al., 1976; Sasak et $a l ., 1977)$ and strongly support a demand for the elucidation of the physiological meaning of this phenomenon. One may speculate that, the presence of different polyisoprenoids should be a result of their specific cellular functions. However, except dolichyl phosphate necessary for functioning in complex biosynthesis of oligosaccharide moiety or glycosylphosphatidylinositol anchors of glycoproteins, common in all eukariots, no function has been attributed and confirmed to other polyisoprenoids.

To answer the question of specificity of metabolic processes utilizing polyisoprenoids several approaches have been used. A wide range 
Table 4. Comparison of polyisoprenoids in seeds and leaves of angiosperms

\begin{tabular}{|c|c|c|}
\hline Species & Prenologues (no of isopren units) ${ }^{a, b}$ & Content (\% of dry weight) ${ }^{\mathbf{c}}$ \\
\hline \multicolumn{3}{|c|}{ Acer rubrum } \\
\hline Seeds & $16, \mathbf{1 7}, 18,19,20$ & 0.003 \\
\hline Leaves & $10,11, \mathbf{1 2}, 13$ & 0.2 \\
\hline \multicolumn{3}{|c|}{ Hippophae rhamnoides } \\
\hline Seeds & $15,16, \mathbf{1 7}, 18,19$ & 0.003 \\
\hline Leaves & $10, \mathbf{1 1}, 1215,16,17,18, \mathbf{1 9}, 20,21,22$, & 0.1 \\
\hline \multicolumn{3}{|c|}{ Fagus silvatica } \\
\hline Seeds & $15,16, \mathbf{1 7}, 18,19$ & 0.003 \\
\hline Leaves & $\mathbf{1 0}, 1114,15, \mathbf{1 6}, 17,18,19,20,21,22,23$ & 0.2 \\
\hline \multicolumn{3}{|c|}{ Quercus rubra } \\
\hline Seeds & $16, \mathbf{1 7}, 18,19$ & 0.006 \\
\hline Leaves & $10, \mathbf{1 1}, 15,16, \mathbf{1 7}, 18,19,20$ & 0.2 \\
\hline \multicolumn{3}{|c|}{ Tilia cordata } \\
\hline Seeds & $16, \mathbf{1 7}, 18,19,20$ & 0.003 \\
\hline Leaves & $\mathbf{1 0}, \mathbf{1 1}, 1216,17,18,19, \mathbf{2 0}, 21,22,23 \ldots 30$ & 0.1 \\
\hline
\end{tabular}

${ }^{\mathrm{a}}$ The numbers refer to the isoprene units of polyisoprenoid alcohols. ${ }^{\mathrm{b}}$ Dominating isoprenologues are indicated in bold. ${ }^{\mathrm{c}}$ Contents calcutated from HPLC peak areas.

of specificity was found, from absolute - only one substrate (with acceptance of the same polyisoprenoid structures with different chain length) to preference of the "wrong" lipid when the enzymes were assayed in vitro towards different polyisoprenoid substrates, Jankowski et al. (1989), Krag (1998). However, recent identification (Gao et al., 2004) of multienzymatic complexes utilizing dolichyl phosphate, located in the intact yeast endoplasmatic reticulum, suggest their increased catalytic specificity. Although cells grown in vitro efficiently take up and utilise radiolabelled polyisoprenoids or their precursors supplied into the medium, even investigation with the use of grown in vitro mutant cells de-

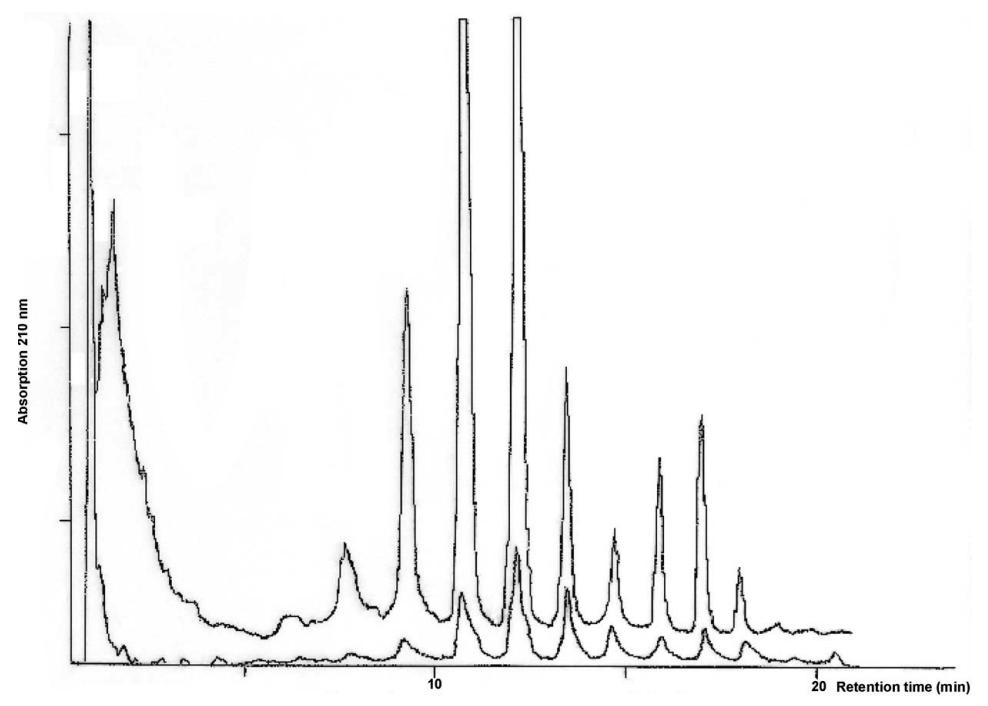

Figure 2. Polyisoprenoid spectra of leaves (upper) and seeds (lower) from T. nucifera. 
fective in the biosynthesis of dolichol from polyprenol does not allow an unequivocal confirmation of utilization of polyprenyl phosphate in the cellular glycoprotein synthesis (Jankowski, 1987; Krag, 1998). Also, the postulated role of dolichol as a cellular substrate for the biosynthesis of dolichyl phosphate in germinating soybean in the presence of high activity of dolichyl phosphate phosphatase is not easy to rationalize (Ravi et al., 1986).

In yeast, the content and polyisoprenoid spectrum depend on the phase of growth and/or starving condition. Induction by stress of a synthesis of the second family of longer dolichols could be a necessary cellular mechanism for the adaptation to new conditions of cultivation (Sato et al., 2001; Szkopinska et al., 2002).

In higher plants, biosynthesis of polyisoprenoids is a very complex system of connections between different cellular compartments and metabolic pathways (Rohmer, 1999). Therefore, the multiple families of polyisoprenoids encountered in plant tissues could be a product of different biosynthetic pathways simultaneously or sequentially active in different conditions of vegetation.

When both types of isoprenoids are present in the extract in appreciable amounts it is possible to separate them by the careful use of simple chromatographic separation methods (Jankowski \& Chojnacki, 1991). However, if a complex mixture of microgram quantities of polyisoprenoids is extracted from the tissue together with large amounts of lipids with similar properties it is necessary to upscale the procedure and to use disposable analytical columns in the multistep purification before the final analysis allowing their separation and estimation of the content of individual lipid (Bamba et al., 2001). Due to the low content of polyisoprenoids in seeds their use as a taksonomic marker is of limited value especially that unequivocal identification of particular member of polyisoprenoid spectrum requires very precise work, disposable vessels and costly instruments.
We are grateful to Professor Tadeusz Chojnacki for inspiration and help in initiation of this work and to Ms. Józefina Hertel for the access to "Collection of Polyprenols".

\section{R E F E R E N C E S}

Bamba T, Fukusaki E, Kajiyama S, Ute K, Kitayama T, Kobayashi A. (2001) The occurrence of geometric polyprenol isomers in the rubber-producing plant, Eucomia ulmoides Oliver. Lipids.; 7: 727-32.

Bamba T, Fukusaki E, Nakazawa Y, Kobayashi A. (2004) Rapid and high-resolution analysis of geometric polyprenol homologues by connected octadecylsilylated monolythic silica columns in high-performance liquid chromatography. J Sep Sci.; 27: 293-6.

Bergamini E, Bizzari R, Cavallini G, Cerbai B, Chiellini E, Donati A, Gori Z, Manfrini A, Parentini I, Signori F, Tamburini I. (2004) Aging and oxidative stress: a role for dolichol in the antioxidant machinery of cell membranes? J Alzheimer's Dis.; 6: 129-35.

Chojnacki T, Vogtman T. (1984) The occurrence and seasonal distribution of $\mathrm{C}_{30}-\mathrm{C}_{60}$-polyprenols and of $\mathrm{C}_{100^{-}}$and similar long-chain polyprenols in leaves of plants. Acta Biochim Polon.; 31: 115-26.

Gao XD, Nishikawa A, Dean N. (2004) Physical interaction between the Alg1, Alg2, and Alg11 mannosyltransferases in the endoplasmic reticulum. Glycobiology.; 14: 559-70.

Hemming FW. (1992) Dolichol: a curriculum cognitionis. Biochem Cell Biol.; 70: 377-81.

Jankowski WJ. (1987) Uptake and metabolism of exogenous polyprenols by animal cells. Chem Scripta.; 27: 5-9.

Jankowski WJ, Chojnacki T. (1991) Long chain polyisoprenoid alcohols in leaves of Capparis species. Acta Biochim Polon.; 38: 265-76.

Jankowski WJ, Palmarczyk G, Krajewska I, Vogtman T. (1989) Specificity of cellular processes and enzymes towards polyisoprenoids 
of different structure. Chem Phys Lipids.; 51: $249-59$.

Jankowski WJ, Kula-Swiezewska E, Sasak W, Chojnacki T. (1994) Occurence of polyprenols and dolichols in plants. J Plant Phys.; 143: 448-52.

Krag SS. (1998) The importance of being dolichol. Biochem Biophys Res Commun.; 243: 1-5

Lindgren BO. (1965) Homologous aliphatic $\mathrm{C}_{30}-\mathrm{C}_{45}$ terpenols in birch wood. Acta Chem Scand.; 19: 1317-26.

Mańkowski T, Jankowski W, Chojnacki T, Franke P. (1976) $\mathrm{C}_{55}$-dolichol: occurrence in pig liver and preparation by hydrogenation of plant undecaprenol. Biochemistry.; 10: $2125-30$.

Radomińska-Pyrek A, Chojnacki T, Pyrek JS. (1979) Fully unsaturated decaprenol from bovine pituitary glands. Biochem Biophys Res Commun.; 86: 395-401.

Ravi K, Rip JW, Carrol KK. (1984) Differences in polyisoprenoid alcohols of mono and dicotyledonous seeds. Lipids.; 19: 401-4.

Ravi K, Rip JW, Carroll K. (1986) Regulation of levels of dolichol and dolichyl phosphate during germination and early development of soybeans. Biochim Biophys Acta.; 875: 626-32.

Rohmer M. (1999) The discovery of mevalonate-independent pathway for isoprenoid biosynthesis in bacteria, algae and higher plants. Nat Prod Rep.; 16: $565-74$.

Rezanka T, Votruba J. (2001) Chromatography of long-chain alcohols (polyprenols) from animal and plant sources. J Chromatogr A.; 936: $95-110$.
Sasak W, Mankowski T, Chojnacki T. (1977) Heterogeneity of $\mathrm{C}_{55}$-polyprenol from leaves of Magnolia campbelli. Chem Phys Lipids.; 18: 199-204.

Sato M, Fujisaki S, Sato K, Nishimura Y, Nakano A. (2001) Yeast Saccharomyces cerevisiae has two cis-prenyltransferases with different properties and localization for their distinct physiological roles in dolichol synthesis. Genes Cells.; 6: 495-506.

Skorupińska-Tudek K, Bieńkowski T, Olszowska O, Furmanowa M, Chojnacki T, Danikiewicz W, Świeżewska E. (2003) Divergent pattern of polyisoprenoid alcohols in the tissues of Coluria geoides: a new electrospray ionization MS approach. Lipids.; 38: 981-90.

Stone KJ, Wellburn AR, Hemming FW, Pennock JF. (1967) The characterization of ficaprenol -10, -11 and -12 from the leaves of Ficus elastica (decorative rubber plant). Biochem J.; 102: 325-30.

Suga T, Ohta S, Nakai A, Munesada K. (1989) Glicynoprenols: novel polyprenols possessing a phytyl residue from the leaves of soybean. J Org Chem.; 54: 3390-3.

Szkopinska A, Świeżewska E, Rytka J. (2002) Induction of the synthesis of an additional family of long chain dolichols in the yeast Saccharomyces cerevisiae. Effect of starvation and ageing. Acta Biochim Polon.; 49: 781-7.

Świeżewska E, Sasak W, Mańkowski T, Jankowski W, Vogtman T, Krajewska I, Hertel J, Skoczylas E, Chojnacki T. (1994) The search for plant polyprenols. Acta Biochim Polon.; 41: 221-60.

Wellburn AR, Hemming FW. (1966) The occurrence and seasonal distribution of higher isoprenoid alcohols in the plant kingdom. Phytochemistry.; 5: 969-75. 